\title{
Hopping Conductance in Molecular Wires Exhibits a Large Heavy- Atom Kinetic Isotope Effect
}

\author{
Quyen Van Nguyen ${ }^{\dagger}$ and C. Daniel Frisbie ${ }^{\dagger, *}$ \\ 'Department of Chemical Engineering and Materials Science, University of Minnesota, Minneapolis, Minnesota, 55455, \\ United States
}

\begin{abstract}
We report a large kinetic isotope effect (KIE) for intramolecular charge transport through pi-conjugated oligophenylene imine (OPI) molecules $>4 \mathrm{~nm}$ in length connected to $\mathrm{Au}$ electrodes. ${ }^{13} \mathrm{C}$ and ${ }^{15} \mathrm{~N}$ heavy-atom substitution on the imine bonds produces a normalized conductance KIE of $\sim 2.7$ per labeled atom in OPI wires, far larger than typical heavy-atom KIEs reported for chemical reactions. In contrast, isotopic labeling of the imine bonds for short OPI wires $<4 \mathrm{~nm}$ does not produce a conductance KIE, consistent with a direct tunneling mechanism expected for short molecules. Temperature dependent measurements on a long $(>4$ $\mathrm{nm}){ }^{15} \mathrm{~N}$-substituted OPI wire and its unlabeled isotopologue reveal that conductance is activated. The conductance results for long wires are thus consistent with multi-step polaron transport and we propose that the exceptionally large conductance KIEs imply a thermally-assisted, through-barrier polaron tunneling mechanism. In general, the observation of large heavy-atom conductance KIEs opens up considerable opportunities for exploring microscopic conduction mechanisms in pi-conjugated molecules.
\end{abstract}

The use of isotopic labeling to examine electrical conduction mechanisms in molecular conductors and semiconductors is relatively rare, ${ }^{1-3}$ perhaps because the effects have generally been expected to be quite small. In principle, however, exploration of conduction isotope effects in molecular systems holds promise for deciphering charge transport mechanisms, ${ }^{4-6}$ including rate limiting steps and transition states, in exact analogy with the study of kinetic isotope effects for determining reaction mechanisms in physical organic chemistry.

Here we report the discovery of a large kinetic isotope effect (KIE) for hopping conduction in nanoscopic pi-conjugated molecular wires connected between $\mathrm{Au}$ electrodes. Our experiments focus on ${ }^{13} \mathrm{C}$-, ${ }^{15} \mathrm{~N}$ - and D-labeled oligophenylene imine (OPI) wires grown from $\mathrm{Au}$ substrates, Figure 1A (see also Figures S1-S3 in Supporting Information), with the number of phenylenes ranging from $n=2-10$, corresponding to molecular lengths of 1-7 nm. Figure $1 \mathrm{~A}$ shows that our labeling studies to date have focused on the imine linkage $(-\mathrm{C}(\mathrm{H})=\mathrm{N}-)$. The OPI wires are synthesized on $\mathrm{Au}$ surfaces beginning with formation of self-assembled monolayers (SAMs) of 4-aminothiophenol followed by sequential 'click-like' condensation reactions with aryl dialdehydes and diamines, as we have reported previously. ${ }^{7-10}$ Isotopic substitution is achieved by using monomers shown in Figure 1B. Monomers 1, 2, and 7-10 are commercially available and 3-6 were synthesized as described in Supporting Information. The syntheses of 27 different ${ }^{13} \mathrm{C}-,{ }^{15} \mathrm{~N}$ and D-substituted OPI wires and 9 unsubstituted versions, along with their corresponding characterization by reflection-absorption infrared spectroscopy (RAIRS), are reported in Figures S4-S6 in Supporting Information.

(A) OPI-7X Molecular Wires

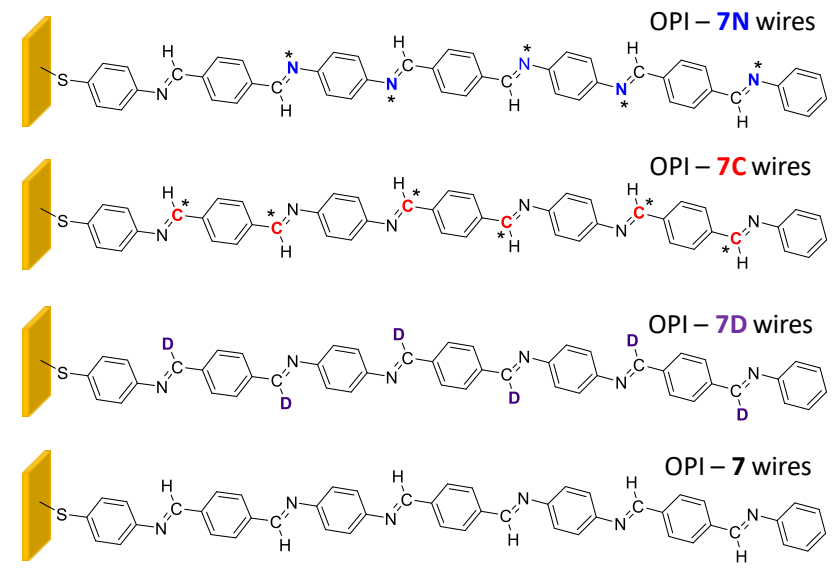

(B) Monomers

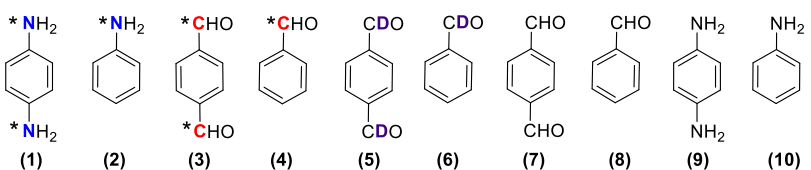

Figure 1. (a) Structures of isotopologues OPI-7N, OPI-7C, OPI$7 \mathrm{D}$, and the unsubstituted OPI-7 parent molecule on Au. The general naming scheme is OPI- $n \mathrm{X}$, where $n=2-10$ is the number of phenylene rings and $\mathrm{X}$ is the isotope. (b) The corresponding monomer units used to synthesize the OPI wires.

To measure the conductance (or resistance) of the isotopically substituted OPI wires, we employed the conducting probe atomic force microscopy (CP-AFM) platform, Figure 2A. ${ }^{7,9-15} \mathrm{Au}$-coated probe tips were brought into soft $(\sim 1 \mathrm{nN})$ contact with the OPI wire films and voltage was swept at the tip while the substrate was grounded. Figure 2B displays the resulting current-voltage $(I-V)$ characteristics on a semi-log scale for OPI-4 and the ${ }^{13} \mathrm{C}-,{ }^{15} \mathrm{~N}-$, and D-labeled versions, OPI-4C, OPI-4N, and OPI-4D, respectively. Differences in average currents are within the error of the measurements, and there is no apparent KIE. We have established previously that for short OPI molecules up to OPI-5, the dominant electrical transport mechanism is direct tunneling, ${ }^{7,9-10,12}$ and in such a situation, a significant KIE is not expected.

Figure $2 \mathrm{C}$ reveals a very different result for the longer oligomers OPI-7, OPI-7C, and OPI-7N. The ${ }^{13} \mathrm{C}$ - and ${ }^{15} \mathrm{~N}$-substituted OPI-7C 
and OPI-7N molecules have significantly lower current than OPI7, by about a factor of 3-5, at all voltages. Similar observations hold for $n=6$ and longer $(n=8-10){ }^{13} \mathrm{C}$ - and ${ }^{15} \mathrm{~N}$-substituted wires, as shown in Supporting Information, Figures S7-S9. However, there is no apparent difference in currents for OPI-7D versus OPI-7. In prior work, we have demonstrated that the mechanism of charge transport in long OPI wires is thermally activated, multi-step hopping in which holes are injected into the OPI pi-systems and driven along the molecules by the externally applied electric field. ${ }^{7}, 9-10,12$ The localized hole is best described as a polaron, i.e., charge and the accompanying geometric distortion of bond lengths and angles in the molecular backbone, Scheme 1. In this situation, transition state theory applies for each intramolecular polaron hop and one expects a KIE..$^{16-25}$ Our observations of KIEs in long molecules ( $n$ $=6-10)$ are consistent with this expectation.

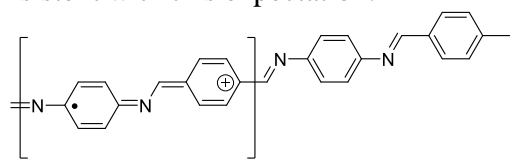

Scheme 1. Representation of a polaron in an OPI oligomer.

Low bias resistance versus molecular length results for all 36 OPI wires that differ in length $(n=2-10)$ and type of substitution are shown in Figure 3A. The tunneling and hopping transport regimes are defined by the stark slope change evident in the resistance data. For short OPI molecules below $4 \mathrm{~nm}$ in which direct tunneling dominates, the resistance increases exponentially with molecular length, as expected. ${ }^{7,9-10,12,26-33}$ There is no KIE in this regime for any of the wires; the resistance versus length behavior is indistinguishable for the ${ }^{13} \mathrm{C}-,{ }^{15} \mathrm{~N}$-, and D-labeled molecules versus the unlabeled versions. For long OPI wires $>4 \mathrm{~nm}$ where the transport mechanism is polaron hopping, large KIEs are evident. In particular, the ${ }^{13} \mathrm{C}$ and ${ }^{15} \mathrm{~N}$-substituted wires have distinctly higher resistances $(R)$ than the unlabeled OPI wires. The slopes of the $R$ vs length trends are also steeper for the ${ }^{13} \mathrm{C}$ and ${ }^{15} \mathrm{~N}$ isotopologues than for the unlabeled versions. Additionally, Figure 3A shows that $\mathrm{H} / \mathrm{D}$ exchange on the imine functionality did not produce a KIE for any OPI wire length.

Conductance KIEs are quantified by the ratio of wire conductances $G(=1 / R)$, which are directly proportional to polaron hopping rates, i.e., $\mathrm{KIE}=G_{\text {light }} / G_{\text {heavy }}=R_{\text {heavy }} / R_{\text {light }}$, where $G_{\text {light }}\left(R_{\text {light }}\right)$ is the conductance (resistance) of the unlabeled molecule and $G_{\text {heavy }}$ ( $R_{\text {heavy }}$ ) corresponds to the heavy-atom isotopologue. For all ${ }^{13} \mathrm{C}$ and ${ }^{15} \mathrm{~N}$-substituted wires in the hopping regime, KIE $>1$, as shown in Figure 3B and Table 1. These conductance KIEs are exceptionally large, ranging from 3-30. Primary KIEs for reactions involving ${ }^{13} \mathrm{C}$-labeled molecules, and to a lesser extent ${ }^{15} \mathrm{~N}$-isotopologues, are documented in solution and gas-phase studies and are generally quite small $\left({ }^{13} \mathrm{C} \mathrm{KIE}<1.01\right)$ near room temperature. ${ }^{34-39}$ However, unlike typical reaction kinetics studies in which single bond breaking or formation rates are the primary focus, the conductance KIEs reported here represent an aggregate of multiple intramolecular polaron transfer events, involving many bonds in the wire backbone depending on the degree of polaron localization. For example, it is evident in Figure 3B that the KIE generally increases as OPI wire length increases, indicating that additional labeled imine bonds reduce the overall hopping conductance in the heavy isotopologues.

To normalize the KIEs we have divided total KIE for each wire by the number of isotope atoms present in the wire (e.g., $n$ - 1 isotope atoms for OPI- $n \mathrm{C}$ wires). The isotope normalized KIEs are also shown in Table 1 and they range from $0.7-3.3$, generally increasing with length. We believe the isotope normalized KIEs for the longest wires (shaded in blue in Table 1) are more reliable than for the $n=6-8$ wires, for two reasons. First, there will be more polaron hopping events in the longest wires and hence a greater number of labeled imine bonds will be sampled. Second, it seems quite likely there are 'end-effects' in the conductance measurements that impact the calculation of the normalized KIE. For example, the normalized KIE for the OPI-6C wires is 0.7 (Table 1), which suggests a rate increase compared to the unlabeled OPI-6, but this is at odds with the total KIE for OPI-6C wires that shows they are 3.6x more resistive than unlabeled OPI-6 wires. This discrepancy is resolved if one presumes that imine bonds closest to the contacts somehow do not contribute to the wire resistance, perhaps because injected charge is initially distributed over several rings. The upshot is that we take the normalized KIEs for OPI-9C, OPI-9N, OPI-10C, and OPI-10N in Table 1 as better values. The approximate mean normalized KIE for these wires is 2.7, which again is an extraordinarily large value compared to typical heavy atom KIEs in chemistry.

(A)
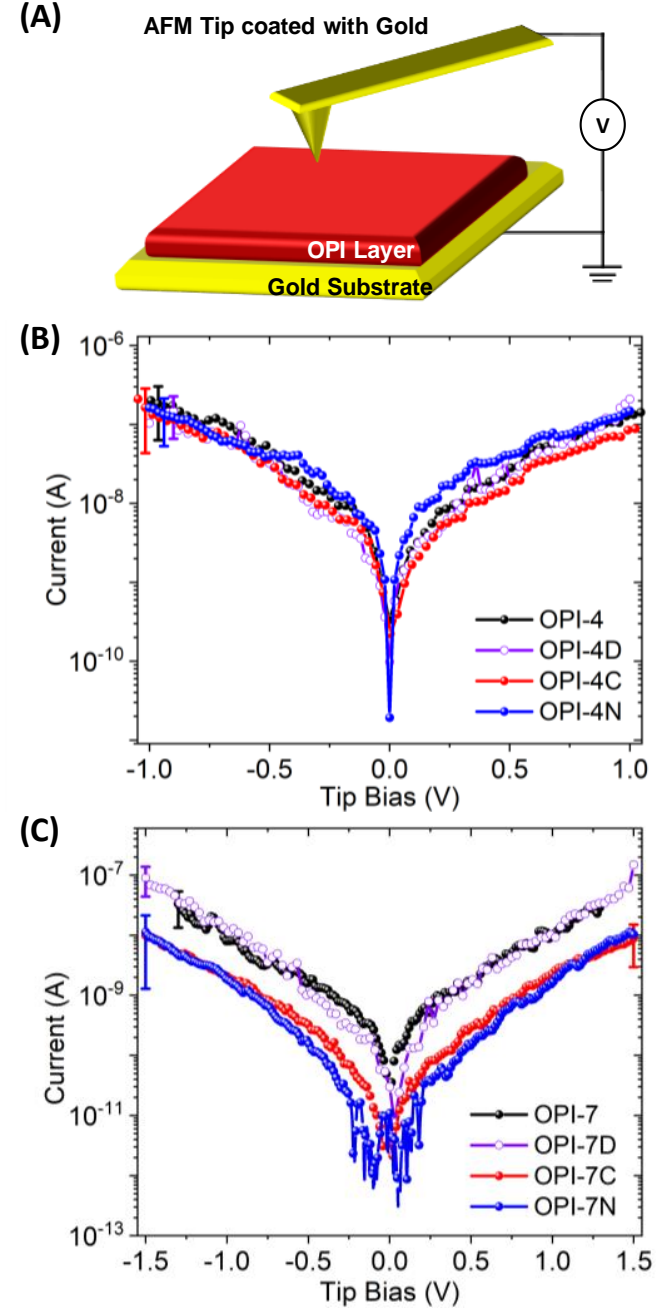

Figure 2. (a) Schematic representation of the CP-AFM setup. (b) Semi-log plots of averaged $I-V$ curves for OPI-4 and isotopologues OPI-4X. (c) Averaged $I-V$ curves for OPI-7 and isotopologues OPI-7X. Each curve is the average of $200 I-V$ traces. The error bars represent one standard deviation.

As noted above, the observation of conductance KIEs for long ${ }^{13} \mathrm{C}$ and ${ }^{15} \mathrm{~N}$-substituted wires is anticipated. Prior quantum chemical calculations by our collaborators for isolated, positively charged OPI wires revealed that the polaron is localized on only a few repeat units such that multiple hops are necessary to traverse the entire wire length. ${ }^{9-10}$ These calculations also indicated that the intramolecular hopping transition states involve simultaneous reduction (flattening) of the dihedral angle between adjacent phenylenes and lengthening of the $-\mathrm{C}=\mathrm{N}$ - (imine) bond. In this scenario, the substitution of heavy isotopes in the imine linkage is expected to produce a KIE as the ground state vibration frequency of the imine bond is 
decreased, leading to a higher barrier and fewer attempted crossings through the transition state per unit time. It is intriguing to consider whether ${ }^{13} \mathrm{C} /{ }^{12} \mathrm{C}$ exchange in the phenyl rings has a similar impact as labeling the imine functionality. Those studies are ongoing. Likewise, we note that the trends in Figure 3B are not perfectly monotonic, i.e., there is some fluctuation in KIE with increasing $n$, and further investigation is necessary to determine the causes. Nevertheless, our current observations verify the key role of the imine bond on the intramolecular polaron hopping rate.
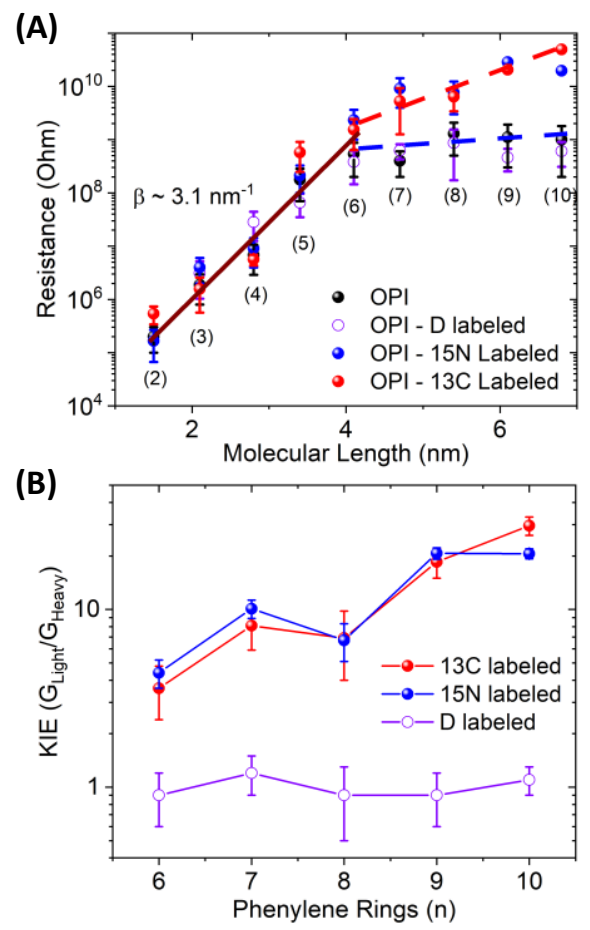

Figure 3. (a) Semi-log plot of low bias $( \pm 0.5 \mathrm{~V})$ resistance versus molecular wire length. Corresponding ring numbers are also indicated. Error bars are one standard deviation. Each point represents the average of $200 I-V$ traces. (b) KIEs for labeled OPI wires versus ring number extracted from the data in (a).

Table 1. Summary of the kinetic isotopic effect for labeled OPI wires. $\mathrm{KIE}=G_{\text {light }} / G_{\text {heavy }}=R_{\text {heavy }} / R_{\text {light }}$. KIE per ${ }^{13} \mathrm{C} /{ }^{15} \mathrm{~N}$ is the KIE divided by the number of heavy isotope atoms per wire.

\begin{tabular}{cccccc}
\hline $\begin{array}{c}\text { Ring } \\
\text { Number }\end{array}$ & ${ }^{13} \mathrm{C}$ & KIE per ${ }^{13} \mathrm{C}$ & ${ }^{15} \mathrm{~N}$ & KIE per ${ }^{15} \mathrm{~N}$ & D \\
\hline 6 & $3.6 \pm 1.2$ & $0.7 \pm 0.2$ & $4.4 \pm 0.8$ & $1.1 \pm 0.2$ & $0.9 \pm 0.3$ \\
\hline 7 & $8.1 \pm 2.2$ & $1.4 \pm 0.4$ & $10.1 \pm 1.2$ & $2.0 \pm 0.2$ & $1.2 \pm 0.3$ \\
\hline 8 & $6.9 \pm 2.9$ & $1.0 \pm 0.4$ & $6.7 \pm 1.6$ & $1.2 \pm 0.3$ & $0.9 \pm 0.4$ \\
\hline 9 & $18.5 \pm 3.5$ & $\mathbf{2 . 3} \pm \mathbf{0 . 4}$ & $20.7 \pm 1.5$ & $\mathbf{2 . 9} \pm \mathbf{0 . 2}$ & $0.9 \pm 0.3$ \\
\hline 10 & $29.6 \pm 3.5$ & $\mathbf{3 . 3} \pm \mathbf{0 . 4}$ & $20.6 \pm 2.5$ & $\mathbf{2 . 5} \pm \mathbf{0 . 3}$ & $1.1 \pm 0.2$ \\
\hline
\end{tabular}

The critical question is why the observed conductance KIEs are so large, far larger than ${ }^{13} \mathrm{C}$ or ${ }^{15} \mathrm{~N}$ KIEs reported in the literature. Computational estimates of the transition state energies for polaron hopping in OPIs are on the order of $100 \mathrm{meV}(10 \mathrm{~kJ} / \mathrm{mol}, 2.5$ $\mathrm{kcal} / \mathrm{mol}$ ) corresponding to the concurrent bond stretching and torsional ring motion in pi-conjugated systems just described. Indeed, we have made initial temperature dependent measurements that reveal conductance activation energies of $200-300 \mathrm{meV}$ for both the heavy atom labeled OPI-7N wire and the OPI-7 unlabeled version (see Figure S10 in Supporting Information). It is clear that thermal activation is important for conduction in both OPI-7N and OPI-7, but it does not appear that activation energy differences can explain the large ${ }^{15} \mathrm{~N}$ KIE $=10$ for the OPI-7N isotopologue at room temperature (Table 1). Furthermore, while heavy atom substitution will decrease the transition state barrier attempt frequency, the small percentage increases in atomic weights for ${ }^{13} \mathrm{C}$ and ${ }^{15} \mathrm{~N}$ substitution are unable to account for the large bond-normalized KIEs. These observations, and the fact noted earlier that typical ${ }^{13} \mathrm{C} \mathrm{KIEs}$ in organic reactions are quite small at room temperature, have suggested to us that a classical over-the-barrier mechanism may be inadequate to explain the large KIEs for intramolecular polaron transport.

An intriguing possibility is that the transport mechanism involves thermally assisted polaron tunneling. ${ }^{18,40-44}$ By polaron tunneling we mean coupled charge and nuclear (e.g., C, N) tunneling, i.e., a simultaneous change in both electronic and nuclear coordinates. Generally speaking, observation of exceptionally large KIEs can be an indicator of nuclear tunneling processes. ${ }^{45-50}$ Nuclear tunneling is well known to play a critical role in the bond shift isomerization of cyclobutadiene $\mathrm{e}^{47,51-53}$ and the umbrella inversion of the ammonia molecule, ${ }^{47,54-56}$ for example, as well as other reactions. ${ }^{34}$, $36-39,47,57-59$ Other authors have also proposed a role for ${ }^{12} \mathrm{C}$-tunneling in polaron transport in molecular semiconductors. ${ }^{40-44}$ For the OPI system, the conditions for nuclear tunneling appear to be met, namely a small barrier height $(\sim 100 \mathrm{meV})$ and a narrow barrier width. The narrow barrier width can be inferred from the polaron hopping transition state, which involves stretching of the imine bond. Changing the imine bond order from two $(\mathrm{C}=\mathrm{N})$ to one $(\mathrm{C}-$ $\mathrm{N}$ ), as implied by the change from aromatic to quinoidal bonding patterns in OPI polarons, ${ }^{7,-10}$ results in a bond length increase of only $\sim 0.2 \AA$, supporting the narrow barrier concept.

However, for a thermally-assisted polaron tunneling mechanism, a crucial question is whether the estimated nuclear tunneling rates are compatible with the rate of charge transfer through the OPI molecules. A second question is whether large KIEs can indeed be predicted, even approximately. Inspection of Figure 2 shows that current levels of $1 \mathrm{nA}$ pass through the metal-OPI-7-metal junctions at $V=0.5 \mathrm{~V}$. From prior work we know that 50-100 OPI-7 molecules are contacted by the CP-AFM probe. ${ }^{8,13,60}$ To make a conservative calculation, we assume that the current passes through on average 10 OPI-7 molecules. This corresponds to $10^{9}$ holes/s per molecule, or a hole transit time per molecule of approximately 1 ns. Approximate rates of nuclear tunneling can be calculated as outlined by Karmakar and Datta in their recent paper on heavy atom tunneling in organic reactions. ${ }^{47}$ Assuming a parabolic barrier for ${ }^{12} \mathrm{C}$ atom tunneling that is $100 \mathrm{meV}(\sim 10 \mathrm{~kJ} / \mathrm{mol})$ in height and $0.2 \AA$ in width, gives a transmission probability $T=5 \times 10^{-4}$. Multiplying $T$ by the $\mathrm{C}=\mathrm{N}$ vibration frequency of $5 \times 10^{13} \mathrm{~s}^{-1}\left(\sim 1600 \mathrm{~cm}^{-1}\right)$, we obtain a ${ }^{12} \mathrm{C}$-tunneling rate of $10^{10} \mathrm{~s}^{-1}$, an extraordinarily large value. Importantly, this tunneling rate is compatible with (faster than) the estimated $10^{9} \mathrm{~s}^{-1}$ rate of hole transfer across the OPI molecules at $V=0.5 \mathrm{~V}$. A similar calculation for ${ }^{13} \mathrm{C}$-tunneling gives $T$ $=3.7 \times 10^{-4}$, yielding a KIE $=1.4$ for a single labeled imine bond. This estimated KIE appears to be within a factor of 2 of our normalized KIE/isotope ( 2.7), and thus is in reasonable agreement given the approximate single particle nature of the calculation.

In summary, we have discovered extraordinarily large heavy atom KIEs associated with intramolecular charge transport in piconjugated oligomers. The results may be consistent with a thermally-assisted, intramolecular polaron tunneling mechanism. Further experiments, for example conductance measurements down to much lower temperatures (e.g., $10 \mathrm{~K}$ ) where activated processes freeze out and tunneling processes dominate, are desirable. ${ }^{4,}$ 18, 40, 42, 44 Additionally, computational analysis will be essential for assessment of the role of nuclear tunneling and for obtaining quantitative agreement between transport experiments and theory, especially in light of the large electric fields involved, which alter the potential energy landscape. It seems clear that the discovery of large heavy-atom KIEs for intramolecular polaron transport - independent of whether heavy-atom tunneling is occurring - opens up 
exciting new opportunities for understanding microscopic conduction mechanisms in molecules. Further, if polaron tunneling effects are confirmed, intramolecular charge transport experiments may also provide a useful platform for examination of heavy-atom tunneling processes generally.

\section{ASSOCIATED CONTENT}

\section{Supporting Information}

The Supporting Information is available free of charge on the ACS Publications website. It includes the chemical structures of all OPI wires, details of OPI wire synthesis and monomer synthesis, reflection-absorption infrared spectra (RAIRS), experimental details on transport measurements, and $I-V$ curves of all labeled OPI wires.

\section{AUTHOR INFORMATION}

\section{Corresponding Author}

C. Daniel Frisbie - Department of Chemical Engineering and Materials Science, University of Minnesota, Minneapolis, Minnesota, 55455, United States; Email: frisbie@umn.edu

\section{Author}

Quyen Van Nguyen - Department of Chemical Engineering and Materials Science, University of Minnesota, Minneapolis, Minnesota, 55455, United States

\section{Notes}

The authors declare no competing financial interest.

\section{ACKNOWLEDGMENT}

The authors acknowledge the financial support of the National Science Foundation (CHE-2003199). Parts of this work were carried out in the Characterization Facility, University of Minnesota, which receives partial support from the NSF through the MRSEC (Award Number DMR-2011401) and NNCI programs (Award Number ECCS-2025124).

\section{REFERENCES}

1. Carlson, K. D.; Williams, J. M.; Geiser, U.; Kini, A. M.; Wang, H. H.; Klemm, R. A.; Kumar, S. K.; Schlueter, J. A.; Ferraro, J. R., The central bond ${ }^{13} \mathrm{C}={ }^{13} \mathrm{C}$ isotope effect for superconductivity in high-Tc $\beta$ (ET) ${ }_{2} \mathrm{I}_{3}$ and its implications regarding the superconducting pairing mechanism. J. Am. Chem. Soc. 1992, 114 (25), 10069-10071.

2. Kato, R.; Aonuma, S.; Sawa, H.; Hiraki, K.; Takahashi, T., Unexpected isotope effect in ${ }^{13} \mathrm{C}$-substituted $(-\mathrm{C} \equiv \mathrm{N})$ molecular conductor (DMe-DCNQI)2Cu (DMe-DCNQI = 2,5-dimethyl-N,N'-dicyanoquinonediimine). Synth. Met. 1995, 68 (2), 195-198.

3. Ren, X.; Bruzek, M. J.; Hanifi, D. A.; Schulzetenberg, A.; Wu, Y.; Kim, C.-H.; Zhang, Z.; Johns, J. E.; Salleo, A.; Fratini, S.; Troisi, A.; Douglas, C. J.; Frisbie, C. D., Negative Isotope Effect on Field-Effect Hole Transport in Fully Substituted ${ }^{13} \mathrm{C}$-Rubrene. Adv. Electron. Mater. 2017, 3 (4), 1700018.

4. Amdursky, N.; Pecht, I.; Sheves, M.; Cahen, D., Marked changes in electron transport through the blue copper protein azurin in the solid state upon deuteration. Proc. Natl. Acad. Sci. U.S.A. 2013, 110 (2), 507.

5. Amdursky, N.; Sepunaru, L.; Raichlin, S.; Pecht, I.; Sheves, M.; Cahen, D., Electron Transfer Proteins as Electronic Conductors: Significance of the Metal and Its Binding Site in the Blue Cu Protein, Azurin. Adv. Sci. 2015, 2 (4), 1400026.

6. Chen, X.; Salim, T.; Zhang, Z.; Yu, X.; Volkova, I.; Nijhuis, C. A., Large Increase in the Dielectric Constant and Partial Loss of Coherence Increases Tunneling Rates across Molecular Wires. ACS Applied Materials \& Interfaces 2020, 12 (40), 45111-45121.

7. Ho Choi, S.; Kim, B.; Frisbie, C. D., Electrical Resistance of Long Conjugated Molecular Wires. Science 2008, 320 (5882), 1482.
8. Demissie, A. T.; Haugstad, G.; Frisbie, C. D., Growth of Thin Anisotropic, $\pi$-Conjugated Molecular Films by Stepwise "Click" Assembly of Molecular Building Blocks: Characterizing Reaction Yield, Surface Coverage, and Film Thickness versus Addition Step Number. J. Am. Chem. Soc. 2015, 137 (27), 8819-8828.

9. Smith, C. E.; Odoh, S. O.; Ghosh, S.; Gagliardi, L.; Cramer, C. J.; Frisbie, C. D., Length-Dependent Nanotransport and Charge Hopping Bottlenecks in Long Thiophene-Containing $\pi$-Conjugated Molecular Wires. J. Am. Chem. Soc. 2015, 137 (50), 15732-15741.

10. Taherinia, D.; Smith, C. E.; Ghosh, S.; Odoh, S. O.; Balhorn, L.; Gagliardi, L.; Cramer, C. J.; Frisbie, C. D., Charge Transport in $4 \mathrm{~nm}$ Molecular Wires with Interrupted Conjugation: Combined Experimental and Computational Evidence for Thermally Assisted Polaron Tunneling. ACS Nano 2016, 10 (4), 4372-4383.

11. Rodriguez-Gonzalez, S.; Xie, Z.; Galangau, O.; Selvanathan, P.; Norel, L.; Van Dyck, C.; Costuas, K.; Frisbie, C. D.; Rigaut, S.; Cornil, J., HOMO Level Pinning in Molecular Junctions: Joint Theoretical and Experimental Evidence. J. Phys. Chem. Lett. 2018, 9 (9), 2394-2403.

12. Sangeeth, C. S. S.; Demissie, A. T.; Yuan, L.; Wang, T.; Frisbie, C. D.; Nijhuis, C. A., Comparison of DC and AC Transport in 1.5-7.5 nm Oligophenylene Imine Molecular Wires across Two Junction Platforms: Eutectic Ga-In versus Conducting Probe Atomic Force Microscope Junctions. J. Am. Chem. Soc. 2016, 138 (23), 7305-7314.

13. Xie, Z.; Bâldea, I.; Demissie, A. T.; Smith, C. E.; Wu, Y.; Haugstad, G.; Frisbie, C. D., Exceptionally Small Statistical Variations in the Transport Properties of Metal-Molecule-Metal Junctions Composed of 80 Oligophenylene Dithiol Molecules. J. Am. Chem. Soc. 2017, 139 (16), 5696-5699.

14. Xie, Z.; Bâldea, I.; Frisbie, C. D., Determination of EnergyLevel Alignment in Molecular Tunnel Junctions by Transport and Spectroscopy: Self-Consistency for the Case of Oligophenylene Thiols and Dithiols on Ag, Au, and Pt Electrodes. J. Am. Chem. Soc. 2019, 141 (8), 3670-3681.

15. Xie, Z.; Bâldea, I.; Frisbie, C. D., Energy Level Alignment in Molecular Tunnel Junctions by Transport and Spectroscopy: SelfConsistency for the Case of Alkyl Thiols and Dithiols on Ag, Au, and Pt Electrodes. J. Am. Chem. Soc. 2019, 141 (45), 18182-18192.

16. Coropceanu, V.; Cornil, J.; da Silva Filho, D. A.; Olivier, Y.; Silbey, R.; Brédas, J.-L., Charge Transport in Organic Semiconductors. Chem. Rev. 2007, 107 (4), 926-952.

17. Oberhofer, H.; Reuter, K.; Blumberger, J., Charge Transport in Molecular Materials: An Assessment of Computational Methods. Chem. Rev. 2017, 117 (15), 10319-10357.

18. Jiang, Y.; Geng, H.; Li, W.; Shuai, Z., Understanding Carrier Transport in Organic Semiconductors: Computation of Charge Mobility Considering Quantum Nuclear Tunneling and Delocalization Effects. J. Chem. Theory Comput. 2019, 15 (3), 1477-1491.

19. Nitzan, A., Electron Transmission Through Molecules and Molecular Interfaces. Annu. Rev. Phys. Chem. 2001, 52 (1), 681-750.

20. Nitzan, A.; Ratner, M. A., Electron Transport in Molecular Wire Junctions. Science 2003, 300 (5624), 1384.

21. Galperin, M.; Ratner, M. A.; Nitzan, A., Molecular transport junctions: vibrational effects. J. Phys.: Condens. Matter 2007, 19 (10), 103201.

22. Galperin, M.; Ratner, M. A.; Nitzan, A., Hysteresis, Switching, and Negative Differential Resistance in Molecular Junctions: A Polaron Model. Nano Lett. 2005, 5 (1), 125-130.

23. Galperin, M.; Ratner, M. A.; Nitzan, A.; Troisi, A., Nuclear Coupling and Polarization in Molecular Transport Junctions: Beyond Tunneling to Function. Science 2008, 319 (5866), 1056.

24. Luo, L.; Choi, S. H.; Frisbie, C. D., Probing Hopping Conduction in Conjugated Molecular Wires Connected to Metal Electrodes. Chem. Mater. 2011, 23 (3), 631-645.

25. Joachim, C.; Ratner, M. A., Molecular electronics: Some views on transport junctions and beyond. Proc. Natl. Acad. Sci. U.S.A. 2005, 102 (25), 8801.

26. Tefashe, U. M.; Nguyen, Q. V.; Lafolet, F.; Lacroix, J.-C.; McCreery, R. L., Robust Bipolar Light Emission and Charge Transport in Symmetric Molecular Junctions. J. Am. Chem. Soc. 2017, 139 (22), $7436-$ 7439.

27. Nguyen, Q. V.; Martin, P.; Frath, D.; Della Rocca, M. L.; Lafolet, F.; Bellinck, S.; Lafarge, P.; Lacroix, J.-C., Highly Efficient LongRange Electron Transport in a Viologen-Based Molecular Junction. J. Am. Chem. Soc. 2018, 140 (32), 10131-10134. 
28. van Nguyen, Q.; Tefashe, U.; Martin, P.; Della Rocca, M. L.; Lafolet, F.; Lafarge, P.; McCreery, R. L.; Lacroix, J.-C., Molecular Signature and Activationless Transport in Cobalt-Terpyridine-Based Molecular Junctions. Adv. Electron. Mater. 2020, 6 (7), 1901416.

29. Hines, T.; Diez-Perez, I.; Hihath, J.; Liu, H.; Wang, Z.-S.; Zhao, J.; Zhou, G.; Müllen, K.; Tao, N., Transition from Tunneling to Hopping in Single Molecular Junctions by Measuring Length and Temperature Dependence. J. Am. Chem. Soc. 2010, 132 (33), 11658-11664.

30. Xiang, D.; Wang, X.; Jia, C.; Lee, T.; Guo, X., Molecular-Scale Electronics: From Concept to Function. Chem. Rev. 2016, 116 (7), 43184440.

31. Bowers, C. M.; Rappoport, D.; Baghbanzadeh, M.; Simeone, F. C.; Liao, K.-C.; Semenov, S. N.; Żaba, T.; Cyganik, P.; Aspuru-Guzik, A.; Whitesides, G. M., Tunneling across SAMs Containing Oligophenyl Groups. J. Phys. Chem. C 2016, 120 (21), 11331-11337.

32. Yan, H.; Bergren, A. J.; McCreery, R.; Della Rocca, M. L.; Martin, P.; Lafarge, P.; Lacroix, J. C., Activationless charge transport across 4.5 to $22 \mathrm{~nm}$ in molecular electronic junctions. Proceedings of the National Academy of Sciences 2013, 110 (14), 5326.

33. Karuppannan, S. K.; Neoh, E. H. L.; Vilan, A.; Nijhuis, C. A. Protective Layers Based on Carbon Paint To Yield High-Quality LargeArea Molecular Junctions with Low Contact Resistance. J. Am. Chem. Soc. 2020, 142 (7), 3513-3524

34. Gómez-Gallego, M.; Sierra, M. A., Kinetic Isotope Effects in the Study of Organometallic Reaction Mechanisms. Chem. Rev. 2011, 111 (8), 4857-4963.

35. Phillips, L. M.; Lee, J. K., Theoretical Studies of Mechanisms and Kinetic Isotope Effects on the Decarboxylation of Orotic Acid and Derivatives. J. Am. Chem. Soc. 2001, 123 (48), 12067-12073.

36. Gable, K. P.; Zhuravlev, F. A., Kinetic Isotope Effects in Cycloreversion of Rhenium (V) Diolates. J. Am. Chem. Soc. 2002, 124 (15), 3970-3979.

37. Snider, M. J.; Reinhardt, L.; Wolfenden, R.; Cleland, W. W. $15 \mathrm{~N}$ Kinetic Isotope Effects on Uncatalyzed and Enzymatic Deamination of Cytidine. Biochemistry 2002, 41 (1), 415-421.

38. Christian, C. F.; Takeya, T.; Szymanski, M. J.; Singleton, D. A., Isotope Effects and the Mechanism of Epoxidation of Cyclohexenone with tert-Butyl Hydroperoxide. J. Org. Chem 2007, 72 (16), 6183-6189.

39. Feilberg, K. L.; Gruber-Stadler, M.; Johnson, M. S.; Mühlhäuser, M.; Nielsen, C. J., 13C, 18O, and D Fractionation Effects in the Reactions of $\mathrm{CH} 3 \mathrm{OH}$ Isotopologues with $\mathrm{Cl}$ and $\mathrm{OH}$ Radicals. J. Phys. Chem. A 2008, 112 (44), 11099-11114.

40. Jiang, Y.; Geng, H.; Shi, W.; Peng, Q.; Zheng, X.; Shuai, Z., Theoretical Prediction of Isotope Effects on Charge Transport in Organic Semiconductors. J. Phys. Chem. Lett. 2014, 5 (13), 2267-2273.

41. Jiang, Y.; Peng, Q.; Geng, H.; Ma, H.; Shuai, Z., Negative isotope effect for charge transport in acenes and derivatives - a theoretical conclusion. Phys. Chem. Chem. Phys. 2015, 17 (5), 3273-3280.

42. Jiang, Y.; Zhong, X.; Shi, W.; Peng, Q.; Geng, H.; Zhao, Y.; Shuai, Z., Nuclear quantum tunnelling and carrier delocalization effects to bridge the gap between hopping and bandlike behaviors in organic semiconductors. Nanoscale Horiz. 2016, 1 (1), 53-59.

43. van der Kaap, N. J.; Katsouras, I.; Asadi, K.; Blom, P. W. M.; Koster, L. J. A.; de Leeuw, D. M., Charge transport in disordered semiconducting polymers driven by nuclear tunneling. Phys. Rev. B $\mathbf{2 0 1 6}$, 93 (14).

44. Asadi, K.; Kronemeijer, A. J.; Cramer, T.; Jan Anton Koster, L.; Blom, P. W. M.; de Leeuw, D. M., Polaron hopping mediated by nuclear tunnelling in semiconducting polymers at high carrier density. Nat. Commun. 2013, 4 (1), 1710.

45. Marcus, R. A., ELECTRON AND NUCLEAR TUNNELING IN CHEMICAL AND BIOLOGICAL SYSTEMS. In Tunneling in Biological Systems, Chance, B.; Marcus, R. A.; Devault, D. C.; Schrieffer, J. R.; Frauenfelder, H.; Sutin, N., Eds. Academic Press: 1979; pp 109-127.

46. Meisner, J.; Kästner, J., Atom Tunneling in Chemistry. Angew. Chem. Int. Ed. 2016, 55 (18), 5400-5413.

47. Karmakar, S.; Datta, A., Heavy-atom tunneling in organic transformations. J. Chem. Sci. 2020, 132 (1), 127

48. Nakanishi, I.; Shoji, Y.; Ohkubo, K.; Ozawa, T.; Matsumoto, K.i.; Fukuzumi, S., A large kinetic isotope effect in the reaction of ascorbic acid with 2-phenyl-4,4,5,5-tetramethylimidazoline-1-oxyl 3-oxide (PTIO') in aqueous buffer solutions. Chem. Commun. 2020, 56 (77), 11505-11507.

49. Schreiner, P. R., Quantum Mechanical Tunneling Is Essential to Understanding Chemical Reactivity. Trends Chem. 2020, 2 (11), 980-989.
50. Castro, C.; Karney, W. L., Heavy-Atom Tunneling in Organic Reactions. Angew. Chem. Int. Ed. Engl. 2020, 59 (22), 8355-8366.

51. Carpenter, B. K., Heavy-atom tunneling as the dominant pathway in a solution-phase reaction? Bond shift in antiaromatic annulenes. J. Am. Chem. Soc. 1983, 105 (6), 1700-1701.

52. Schoonmaker, R.; Lancaster, T.; Clark, S. J., Quantum mechanical tunneling in the automerization of cyclobutadiene. J. Chem. Phys. 2018, 148 (10), 104109

53. Huang, M. J.; Wolfsberg, M., Tunneling in the automerization of cyclobutadiene. J. Am. Chem. Soc. 1984, 106 (14), 4039-4040.

54. Park, Y.; Kang, H.; Field, R. W.; Kang, H., The frequencydomain infrared spectrum of ammonia encodes changes in molecular dynamics caused by a DC electric field. Proc. Natl. Acad. Sci. U.S.A. 2019 , 116 (47), 23444.

55. Dennison, D. M.; Uhlenbeck, G. E., The Two-Minima Problem and the Ammonia Molecule. Phys. Rev. 1932, 41 (3), 313-321.

56. Rauk, A.; Allen, L. C.; Mislow, K., Pyramidal Inversion. Angew. Chem., Int. Ed. Engl. 1970, 9 (6), 400-414.

57. Michel, C. S.; Lampkin, P. P.; Shezaf, J. Z.; Moll, J. F.; Castro, C.; Karney, W. L., Tunneling by 16 Carbons: Planar Bond Shifting in [16]Annulene. J. Am. Chem. Soc. 2019, 141 (13), 5286-5293.

58. Ertelt, M.; Hrovat, D. A.; Borden, W. T.; Sander, W., Heavyatom tunneling in the ring opening of a strained cyclopropene at very low temperatures. Chem. Eur. J. 2014, 20 (16), 4713-20.

59. Zuev, P. S.; Sheridan, R. S.; Albu, T. V.; Truhlar, D. G.; Hrovat, D. A.; Borden, W. T., Carbon Tunneling from a Single Quantum State. Science 2003, 299 (5608), 867.

60. Demissie, A. T.; Haugstad, G.; Frisbie, C. D., Quantitative Surface Coverage Measurements of Self-Assembled Monolayers by Nuclear Reaction Analysis of Carbon-12. J. Phys. Chem. Lett. 2016, 7 (17), 3477-3481. 